\title{
Panel Summary
}

\section{Towards excellence in anaesthesia}

\author{
Participants \\ Leo Strunin MD FFARCS FRCPC (Chairman) \\ Department of Anaesthesia, Foothills Hospital at the \\ University of Calgary, Calgary, Alberta \\ Jay B. Fortest MB CH B FFARCS FRCPC \\ Department of Anaesthesia, McMaster University. \\ Hamilton, Ontario \\ (Assessing the risk of anaesthesia) \\ John $\mathrm{N}$. Lunn MD FFARCS \\ Department of Anaesthetics, University of Wales \\ College of Medicine, Cardiff, Wales \\ (Mortality studies in the United Kingdom) \\ Burton S. Epstein MD \\ Department of Anesthesiology, The George Washington \\ University Medical Center, Washington, D.C. \\ (Standards of care in anesthesia) \\ Peter G. Duncan MD FRCPC \\ Depariment of Anaesthesia, University of \\ Saskatchewan, Saskatoon, Saskachewan \\ (Standards of practice - the Canadian experience) \\ Jan M. Davies MSC MD FRCPC and J. Edwin Boyd PH D \\ Departments of Anaesthesia and Psychology, Foothils \\ Hospital at the University of Calgary, Calgary, Alberta \\ (Selection and training of anaesthetic residents)
}

\section{Introduction}

The application to everyday endeavour of the concept of excellence has been promulgated by Tom Peters, ${ }^{1,2}$ who has explored in the world of business those factors which distinguish, in his view, the mundane from the successful. Excellence, quality assurance, standards of care and safety are current "buzz words" in anaesthetic practice, but none of us sets out to give poor or unsafe anaesthetics! Nevertheless there are problems, as evidenced by simple observation and by the inclusion of anaesthesia, anywhere in the world, in the higher risk categories when it comes to

Based on presentations given at the 1987 Annual Meeting of the Canadian Anaesthetists' Society in Calgary, Alberta.

Address correspondence to: Dr. Strunin. malpractice insurance. The symposium was divided into three parts: current problems, anaesthetic standards and the concepts of risk mangement and recruitment and training of residents.

Dr. Forrest described the International Multicenter Study of General Anaesthesia which comprised some 17,000 patients randomly assigned to receive one of four of the most commonly used anaesthetic agents: enflurane, fentanyl, halothane, isoflurane. Patients were followed postoperatively and 67 outcomes were recorded as they occurred. Dr. Forrest particularly emphasized the cardiovascular outcomes. He concluded that there were important differences between the anaesthetic drugs. However, these differences should be combined with analysis of pre-existing risk factors when selecting a general anaesthetic if detrimental outcomes are to be avoided.

Dr. John Lunn reviewed mortality studies in the United Kingdom. He pointed out that until very recently these studies, like the majority world-wide, have only used death as an end-point and have been confined to assessment of anaesthetic causes only; the surgical component being ignored. Dr. Lunn then went on to describe the methodology used in the Confidential Enquiry into Peiroperative Death (CEPOD) which assessed both the surgical and anaesthetic contributions (see Table 1 Mortality Studies in the United Kingdom) to any death occurring within 30 days of operation in the study population of some 555,000 operations.

Dr. Burton Epstein presented the way in which the American Society of Anesthesiologists (ASA) was developing standards of care. He chose as his example the recent publication by the ASA of "Standards for Basic Intra-Operative Monitoring. " Dr. Epstein indicated that it was most important that organizations such as the ASA be active rather than reactive with regard to setting standards. Otherwise external groups, which were already attempting to regulate the practice of anaesthesia, would supersede professional organizations. Analysis of medicolegal cases indicates that undetected hypoxia, with an adverse outcome, is the major cause of high-cost law suits. Although there is now a major movement towards the universal use of pulse oximeters and other expensive 
analytical methods of determining oxygenation, Dr. Epstein stressed that "direct observation of the patient with adequate illumination and exposure" was equally important.

Dr. Peter Duncan provided a comprehensive and provocative picture of the specialty of anaesthesia and its standards in Canada. He first reviewed "The Guidelines to the Practice of Anaesthesia in Canada" pointing out certain deficiencies, including: the lack of legal teeth, delays in revision and the cost implications of the guidelines. This latter led him into a spirited asscssment of the political realities of health funding in Canada. Dr. Duncan then took the provision of obstetrical analgesia as a current example of the difficulties of providing quality care in an area of chronic underfunding and widespread lack of appreciation of the necessity for anaesthetic services; it is particularly depressing that the Canadian Medical Association appeared to condone the situation in its recent Report on Obstetrical Care in Canada.

Dr. Jan Davies, working with Dr. Ed Boyd, outlined the assessment methods of their ongoing study of the recruitment and training of residents in the 16 anaesthesia program across Canada. In addition, the characteristics of the programs and the directors will be asssessed. A battery of tests to evaluate residents entering programs in July 1987 will follow their progress over the next four years.

All the speakers emphasized the need to maintain the momentum of review of anaesthetic practice if we are to achieve excellence in the future. Perhaps the words of Henry K Beecher (1941) commenting on the death of Hannah Greener in Winlayton, County Durbam, England on the 28th January 1848 following an anaesthetic with chloroform are apposite. "Judging from the many papers written (by anesthesiologists) on the subject of clinical statistics it would seem that such compilation is one of their major occupations. I have said before, and I repeat, one's faith in the value of such studies will be considerably weakened by finding that what purport to be statistical analyses have in all but a few cases failed to take into account the most rudimentary requirements of statistical analysis. The casualness with which statistical analysis is so often undertaken does not alter the fact that it is involved, time-consuming and without value unless the fundamental laws of statistics are vigorously observed. As I have commented before, it is unhappily true that statisticians and their statistics are looked apon with suspicion by the rest of the world. Statistical methods are no substitute for common sense; on the other hand, some use of such methods is necessary if common sense is to be preserved in the handling of quantities of data."

\section{References}

1 Peters $T$, Waterman $R H \mathrm{Jr}$. In Search of Excellence.

New York: Random House, 1983.

2 Peters $T$, Austin N. A Passion for Excellence. New York: Random Housc, 1985.

\section{Assessing the risk of anaesthesia}

Outcomes in the context of the complications or side effects of anaesthesia are the consequence of actions that may be physical, pathophysiologic or pharmacologic. For cxample, coma in a patient following anacsthesia could be the result of prolonged action or excessive dose of the drugs used to produce anaesthesia, or it could be the result of severe oxygen deprivation from an incorrectly placed endotracheal tube. Interpretation of the clinical importance of outcomes requires knowledge of nomality, established diagnostic criteria, measurement of frequency, analysis of factors that change risk, and assessment of prognostic consequence as well as influence of trcatment and cause.

The quality of evidence as well as the appropriateness of interpretation are very important whenever clinical decisions are made. It is essential that prior to interpretation of data there is confidence in their accuracy and completeness. All occurrences of defined outcomes must be recorded no matter how trivial these may seem to the observer. This fact alone has made several previous studies of outcome so seriously flawed as to be valueless.

The aim of the International Multicenter Study of General Anaesthesia was to determine if there were any differences of clinical importance among the four drugs used most commonly for general anaesthesia. A randomised comparative clinical trial was conducted in 17,201 patients at 15 University Affilitated Teaching Hospitals from the following centres: The University of Calgary, McMaster University, The Mayo Clinic, The Medical College of Wisconsin, The University of California San Francisco, The University of Pennsylvania, and The University of Pittsburgh.

Each patient was seen preoperatively and a detailed assessment made of disease and current therapy. $\mathrm{Pa}$ tients were then randomly assigned to receive one of the four anaesthetic drugs according to preset protocols in 